\title{
A fatal case of electrocution with unique forensic radiological postmortem findings
}

\author{
Rilana Baumeister $^{1}$ - Sabrina Mauf ${ }^{1}$ Patrick Laberke ${ }^{1}$ - Anais Krupp ${ }^{1}$. \\ Michael J. Thali ${ }^{1} \cdot$ Patricia M. Flach ${ }^{1}$
}

Accepted: 14 September 2015/Published online: 5 October 2015

(C) Springer Science+Business Media New York 2015

\begin{abstract}
Purpose Documentation of forensic radiological observations in postmortem imaging of electrical injuries is not common yet and is less prevalent compared to other forms of burn injuries. However, electrical injuries have high morbidity and mortality. The objective of this case report was the visualization and evaluation of unique electrocution-related injuries by postmortem contrast and non-contrast enhanced imaging compared to a forensic autopsy.

Methods Forensic imaging included whole-body postmortem computed tomography (PMCT), PMCT-angiography (PMCTA), postmortem magnetic resonance tomography (PMMR), and PMMR-angiography (PMMRA). Initial external inspection and subsequent autopsy were performed. Results Imaging results revealed intestinal mucosal pathologies, particularly of the gastric vascular integrity and remarkable rhabdomyolysis of the striated muscles of the extremities. Furthermore, PMCT and PMCTA revealed a hepatic lesion with perihepatic free fluid. The results from external inspection and autopsy correlated to the well-known pathologies of electrocution in the course of a high-voltage incident.

Conclusion Postmortem imaging visualized electrocution-related injuries and aided substantially in the medicolegal investigation. These findings, particularly of the rhabdomyolysis in magnetic resonance tomography, may support the future image interpretation of cases with electrical injuries-in the living and the deceased.
\end{abstract}

Rilana Baumeister

rilana_baumeister@hotmail.de;

Rilana.Baumeister@irm.uzh.ch

1 Institute of Forensic Medicine, Forensic Medicine and Imaging, University of Zurich, Winterthurerstrasse 190/52, 8057 Zurich, Switzerland
Keywords Forensic imaging - PMCT · PMMR . Rhabdomyolysis · Electricity · Angiography

\section{Introduction}

Electrical injuries are less prevalent compared to other forms of burn injuries; however, this type of injury is considered one of the most devastating due to high morbidity and mortality (3-15\%) [1-3]. Saukko and Knight [4] reported that high tension voltage $(>1000 \mathrm{~V})$ causes more fatal injuries than low tension voltage $(<500 \mathrm{~V})$.

Often in cases of electrocution, the hands are in contact with electricity, and the body is grounded through the feet. This situation usually creates a passage of the current across the heart, resulting in ventricular fibrillation terminating in asystole [5, 6] and, rarely, in central regulatory arrest $[4,6]$.

Skeletal muscle spasms are known as "hold-on" effects, which make the release of a victim almost impossible while the current still flows [4]. High-voltage electrical trauma frequently results in extensive and scattered destruction of skeletal muscle due to rhabdomyolysis along the current path [7]. Rhabdomyolysis is one of the potentially lifethreatening consequences seen in surviving victims.

Further typical electrocution pathologies are skin lesions, and organ and muscle damage as the current passes through the skin, producing boiling and electrolysis of tissue fluids. High voltage currents may also result in bone destruction [6].

Electric marks (Joule burns) are considered diagnostic for contact with electricity-occasionally with a distinctive pattern-and are detected at the point of entry of the current, whereas an exit injury may appear as a split or a laceration type skin injury. 
Postmortem computed tomography (PMCT) is currently an established forensic imaging tool; however, the radiological postmortem findings of an electric fatality are not well known.

The objective of this case report is the visualization and evaluation of unique electrocution related injuries by postmortem contrast and non-contrast enhanced imaging compared to a forensic autopsy.

\section{Case history}

Passersby observed a 33-year-old, drunken male on top of the roof of a standing train located in a train station. The subject raised his hands and placed himself in contact with a 15,000 voltage current line with both hands. In the course of this electrical impact, he was hurled from the roof of the train and sustained a fall onto the train platform. Immediate cardiopulmonary resuscitation was unsuccessful.

A medico-legal examination into the death was ordered by the local prosecutor.

\section{Materials and methods}

Forensic imaging included whole-body postmortem computed tomography (PMCT), PMCT-angiography (PMCTA), postmortem magnetic resonance tomography (PMMR), and PMMR-angiography (PMMRA). Native PMCT was performed first, PMMR second, and contrast media was applied afterward. The postmortem contrast medium was a mixture of a water-soluble contrast medium and polyethylene glycol as a contrast-agent dissolver [8].

Before and after postmortem imaging, external inspection of the body and autopsy were performed by forensic pathologists.

\section{PMCT and PMCTA}

The forensic institute used a dedicated, on-site dual-source PMCT scanner (Flash Definition, Siemens, Forchheim, Germany). The routine scan included a whole-body PMCT scan protocol without contrast media. The imaging parameters used followed those recommended by recent literature [8, 9]: tube voltage $120 \mathrm{kVp}$; variable mAs values (reference $400 \mathrm{mAs}$ ) using automatic dose modulation software (CARE dose 4D, Siemens, Forchheim, Germany) and $128 \times 0.6 \mathrm{~mm}$ slice collimation.

PMCTA was performed via unilateral access to the femoral blood vessels. Separate perfusion of the arterial and venous systems was established using a modified heart-lung machine. The arterial injection of the contrast media mixture was performed first, followed by venous injection. A detailed protocol is described in the literature [9].

Images were reconstructed with soft and hard kernels, the corresponding windows and dedicated anatomically aligned reconstructions.

\section{PMMR and PMMRA}

Images were acquired on a dedicated 3 Tesla PMMR (Achieva 3.0 T, Philips Healthcare, Best, Netherlands). A XL-Torso coil was used. Unenhanced PMMR included coronal $5 \mathrm{~mm}$ whole-body sequences (STIR, TR: 10,325, TE: 70; T1 FFE, TR: 254, TE: 2.3; T2 TSE, TR: 3652, TE: 76) and $5 \mathrm{~mm}$ axial thorax and abdomen sequences (STIR TSE, TR: 6591, TE: 60; T1 TSE, TR: 667, TE: 7.12; T2 TSE, TR: 4715, TE: 100), as well as axial thinner sliced (3 mm) stacks of the abdomen (T1 TSE, TR: 734, TE: 6.39; T2 TSE, TR: 5171, TE: 60; STIR, TR: 5864, TE: 60; T2* FFE, TR: 1659 , TE: 16.1, PD TSE, TR: 1800, TE: 25) and a coronal STIR sequence ( $3 \mathrm{~mm}$, TR: 4183, TE: 60).

PMMRA included fat-saturated axial, sagittal, and coronal $3 \mathrm{~mm}$ sequences in T1 (TR: 552-710 and TE: 5.896.39) and an additional axial hemosequence of the abdomen (Ven_BOLD, $1.6 \mathrm{~mm}$, TR: 14.4, TE: 20.3).

\section{Image analysis}

A radiology resident and a board-certified radiologist who were experienced in postmortem forensic imaging performed the reporting, postprocessing and analysis using a multimodality reading solution (Syngo.via, Version VA30A, Siemens, Medical Solutions, Erlangen, Germany).

\section{Results}

\section{Imaging findings}

\section{PMCT and PMCTA}

Routine PMCT revealed minor, hypodense free fluid periand infrahepatically (Fig. 1a). PMCTA showed slight contrast media extravasation subhepatically according to a small lesion of the right lobe of the liver (Fig. 1b, c).

PMCTA displayed intense mucosal enhancement of the gastrointestinal tract (particularly gastric and small bowel mucosal enhancement) (Fig. 1b). This enhancement was intensified in the venous phase, with subsequent increased density of the stomach contents correlating to intraluminal contrast media extravasation (Fig. 2).

Soft tissue and bone destruction was visible (correlating to external inspection), particularly at the current's entry and exit locations (Figs. 3, 4). Additionally, a traumatic 

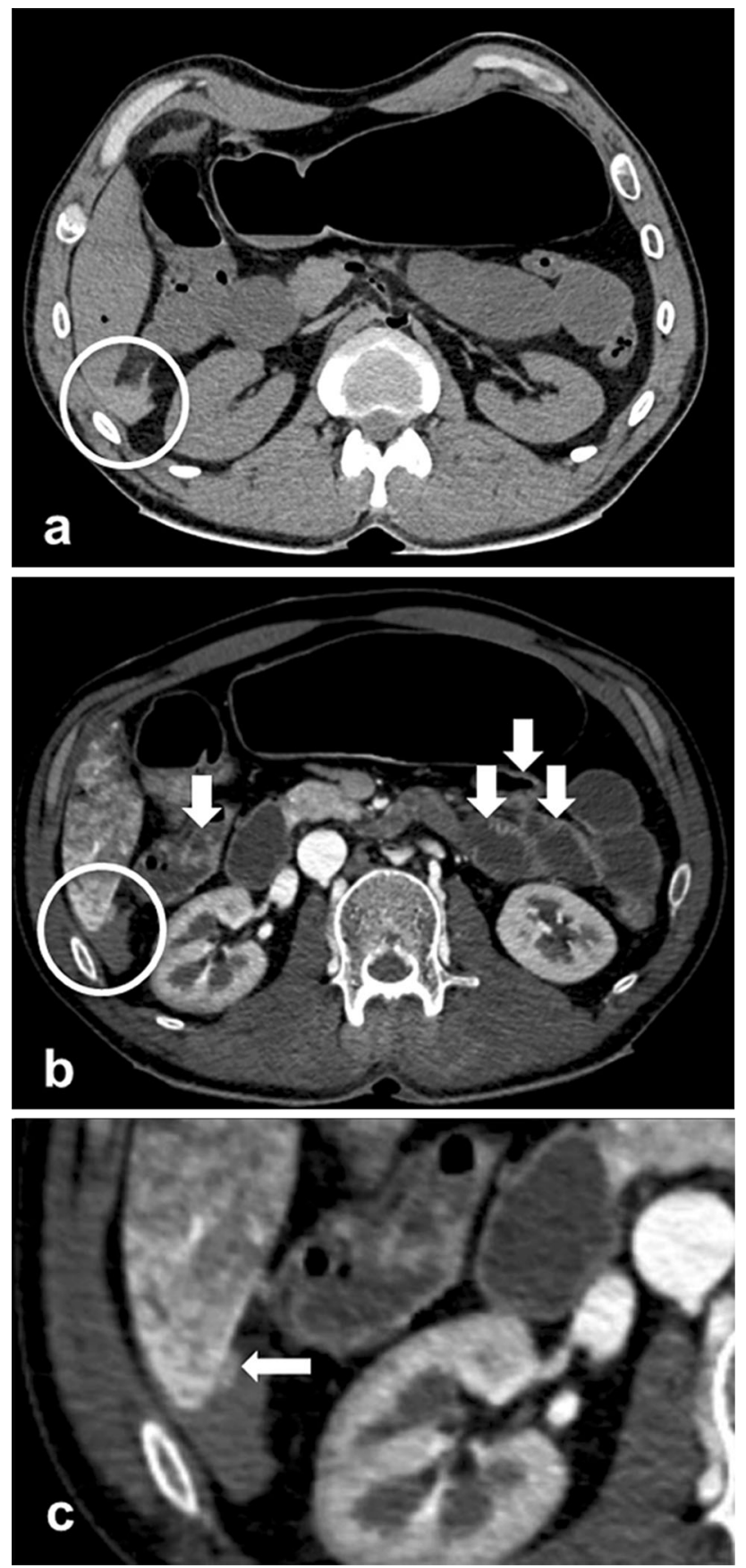

Fig. 1 a Routine axial PMCT of the abdomen at the level of the lower right liver lobe. This image shows slight hyperdense peri- and subhepatic free fluid (circle). b Axial PMCTA (venous injection) of the abdomen at the level of the lower right liver lobe. This image shows discrete subhepatic contrast media extravasation (circle) into the peri- and subhepatic free fluid collection. This finding is suggestive of a liver fissure, which was not detectable macroscopically during autopsy. Note the intense contrast media enhancement of the intestinal mucosa (marked by arrows) in the course of heat or electrically induced mucosal defects. c Detailed view of the contrast extravasation (arrow) caudal to the right lower lobe
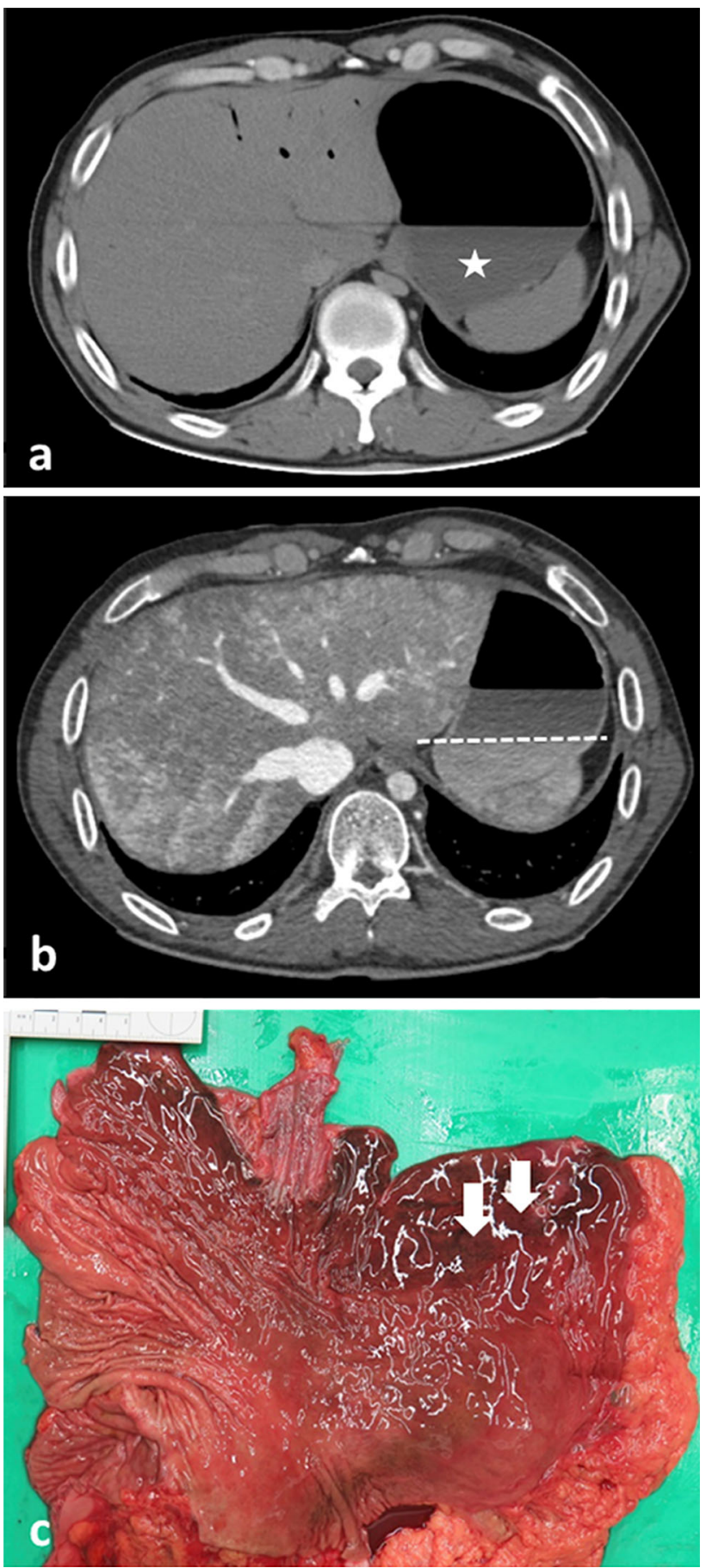

Fig. 2 a Axial PMCT of the abdomen at the level of the liver and stomach. Homogeneous, hypodense content within the stomach (asterisk) without any type of sedimentation. b Axial PMCTA (venous injection) of the abdomen at the level of the liver and stomach. A distinct sedimentation line (dashed line) was detectable with contrast media. The fluid level was due to intraluminal contrast extravasation-attributable to heat or an electrically induced mucosa defect. c Autopsy specimen image of the stomach shows discrete reddish dotted hemorrhage (marked by arrows) 
proximal dislocated fracture of the left femur was reported which was most likely caused by the fall (Fig. 3c). The imaging also indicated edema of the brain (despite normal postmortem swelling of the brain parenchyma) and lung.

\section{PMMR and PMMRA}

Unenhanced PMMR and contrast media-enhanced PMMRA displayed distinct increased signal on fat-saturated short tau inversion recovery (STIR) sequence images within the skeletal muscles (Figs. 3d, 5a-c), particularly in the proximal lower limbs due to general edema.
The perihepatic fluid with subhepatic contrast media extravasation, mucosal enhancement and gastric contrast leakage observed in the CT sections were confirmed. The skin lesion correlating to a Lichtenberg figure was not detectable.

\section{External inspection}

The decedent sustained multiple facial and cranial soft tissue defects as well as superficial abrasions at the torso and the extremities (Fig. 3a, b). Both hands and feet displayed severe tissue damage with destruction down to the bones (Fig. 4).
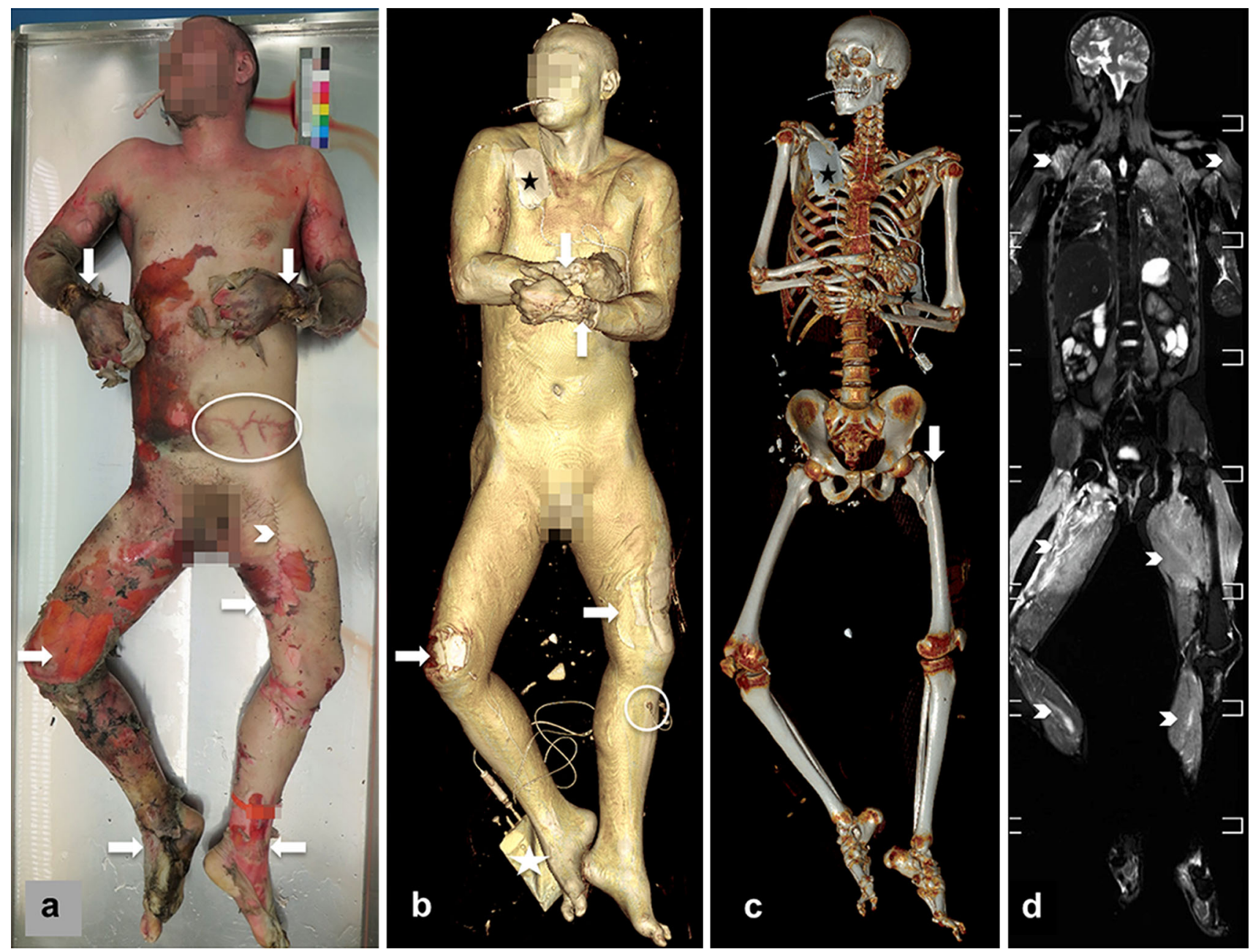

Fig. 3 Frontal whole-body visualization by different modalities. a Autopsy image shows superficial and deep soft tissue defects at the extremities (arrows) and a so-called Lichtenberg figure (ellipse) caused by electrocution. Note the sutured incision on the left groin due to PMCTA before autopsy (arrowhead). The endotracheal tube remains left in place, whereas the tibial access on the left (Fig. 3b) and the defibrillation pads (Fig. 3b, c) are already removed. b PMCT, volume rendering technique. This 3-dimensional reconstruction displays the skin and soft tissue. Note the destruction of the soft tissue (arrows) partially down to the bones, which was induced by electricity and which correlated to the autopsy image. Note the tibial emergency access on the left (circle) with the infusion bag (white asterisk) as well as the defibrillation pad (black asterisk). The endotracheal tube and all other medical devices are left in place for imaging to detect potential malposition. $\mathbf{c}$ This 3-dimensional volume rendering displays the bones and the proximal fracture of the femur (arrow) most likely due to the fall from the train. Note the external defibrillation pads (asterisks). d Coronal whole-body PMMR, short tau inversion recovery (STIR) sequence. Diffuse general muscle edema is shown by hyperintense signal within the skeletal musculature (arrowheads) caused by electrocution-induced rhabdomyolysis 

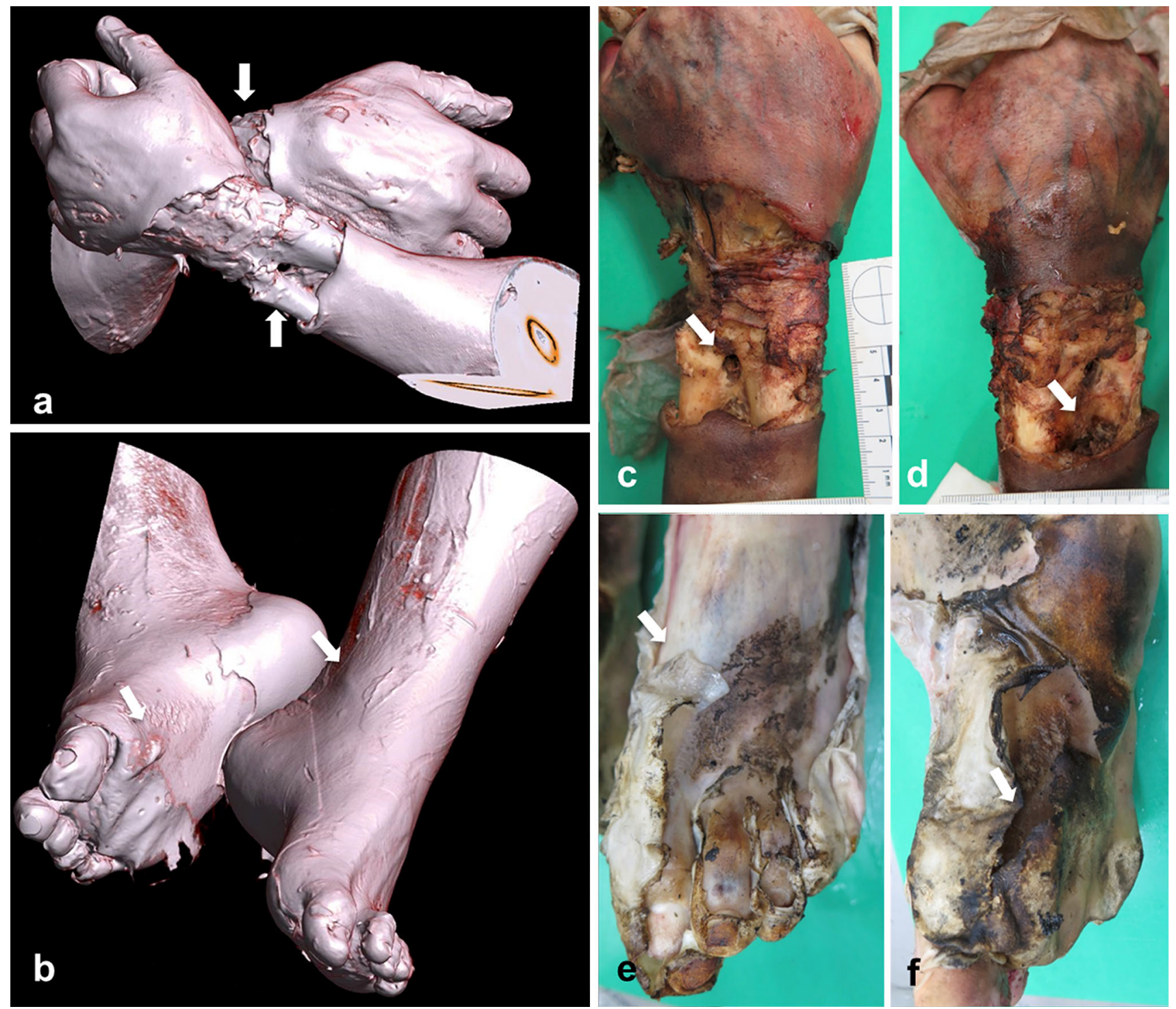

Fig. 4 a PMCT, 3-dimensional reconstruction of both hands and wrists. Anterior view. b PMCT, 3-dimensional reconstruction of both feet. Antero-caudal view. c Autopsy image of the left hand. d Autopsy image of the right hand. e Autopsy image of the left foot. f Autopsy

External inspection showed a reddish, fern-like patterned mark on the skin (a Lichtenberg figure, Fig. 5d) between the lower abdomen and the left hip and an additional white ring with an umbilicated center at the lower back (Fig. 5e). No deformity correlating to fractures were detected by external inspection.

\section{Autopsy}

The autopsy confirmed edema of the brain and lungs, and signs of heat induction in the liver. Macroscopically, no defects of the liver capsule or of the parenchyma were observed. The mucosa of the gastrointestinal tract showed few red dotted bleedings.

image of the right foot. All images display a detailed view of entry and exit wounds on the hands and feet. Note the deep-focused defects at the entry points of both wrists (arrows) and the extensive defects on the exit points of the feet (arrows)

The manner of death was unnatural and in consent compatible with a suicide. The cause of death was electrocution leading to cardiac arrest. Indications for third party interference could not be found.

\section{Discussion}

This case report displays how postmortem imaging supports forensic investigation with unique forensic radiological findings of electrocution and its related injuries.

PMCT displays liver lesions and mucosal damage of the stomach, PMMR detects general edema of skeletal muscles. 

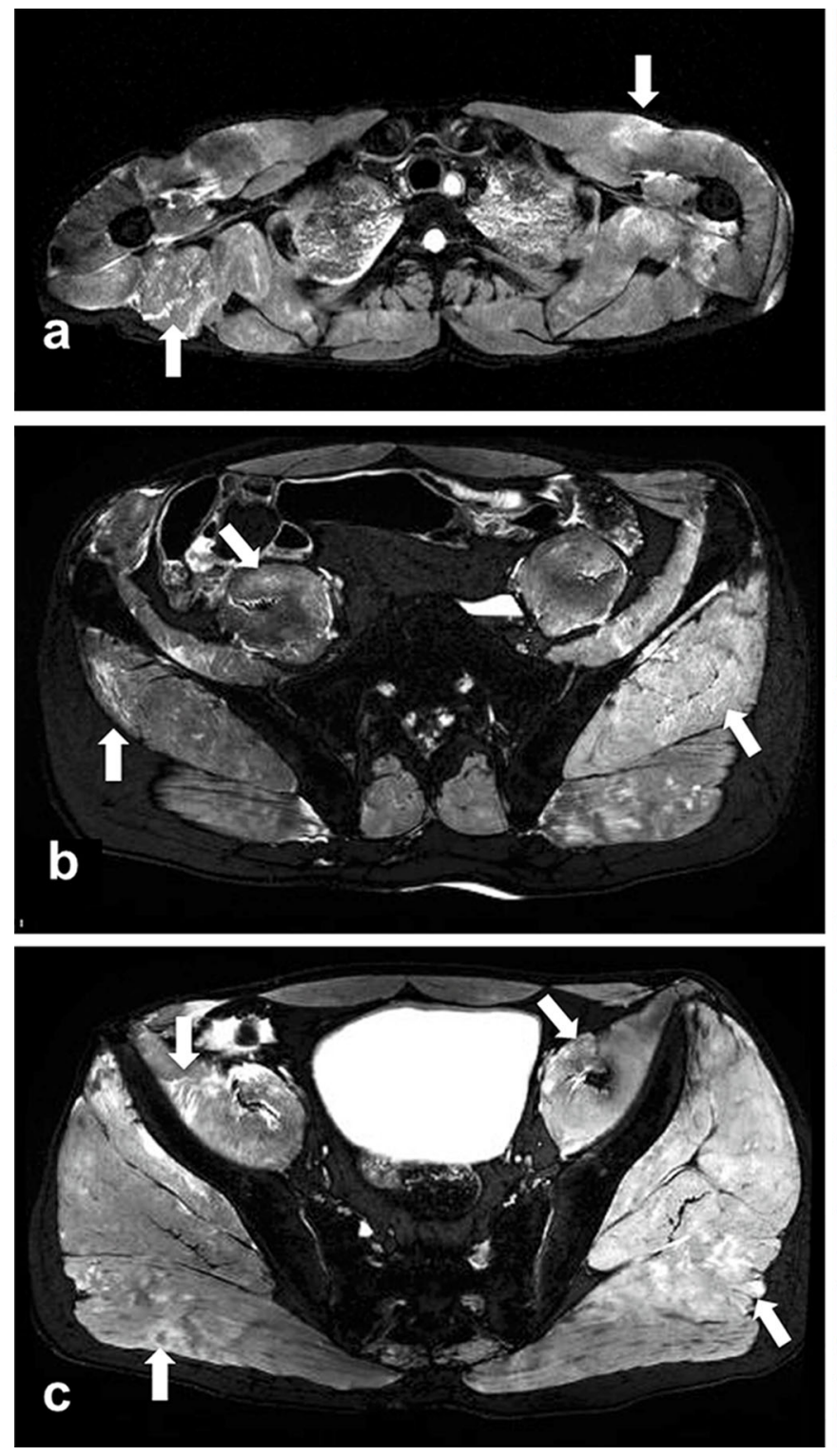

Fig. 5 a-c Axial PMMR, STIR sequence. a Shoulder girdle musculature and the upper thoracic aperture with diffuse intra-musculature edema and rhabdomyolysis due to electrocution (arrows). b Pelvic musculature. Note the high signal within the musculature of the gluteal muscles and the musculus psoas (arrows) due to non-traumatic rhabdomyolysis. c Pelvic musculature caudal to b. Extensive edema is observed in the iliopsoas muscles and the gluteal musculature

Magnetic resonance imaging plays an important role in diagnosing muscle defects and disorders in clinical practice $[10,11]$. The potential use of (contrast-media-enhanced) magnetic resonance imaging to visualize edema in muscles, particularly in high-voltage electrical injuries, has been described in the literature [10-12].

In this case, rhabdomyolysis occurs due to a high voltage fatality and could be visualized by PMMR with generalized skeletal muscle edema. Myonecrosis was not visible by external inspection or by autopsy. Shetty et al.
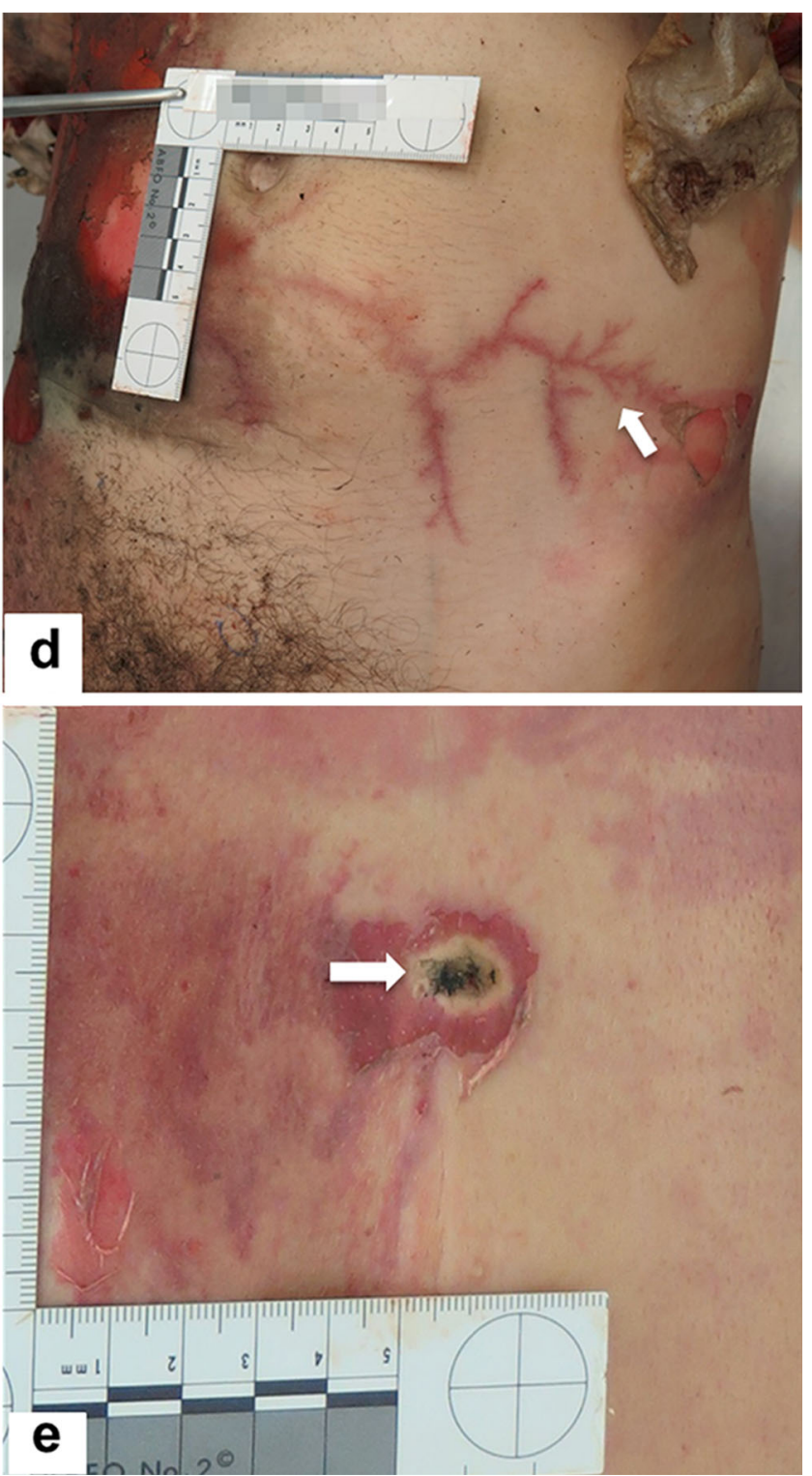

(arrows). d Detailed autopsy image of the left lower abdominal quadrant with an electricity-induced fern-like patterned mark on the skin - the so-called Lichtenberg figure (arrow, also observed on Fig. 3a). e Detailed autopsy image of the back of the body with an additional exit wound (arrow). Both cutaneous marks are only detected by external inspection

[5] and Vale [13] report that non-traumatic rhabdomyolysis may be caused by a direct insult to the cell membrane with the accumulation of abnormal metabolites, e.g., electrical burns or heat shock.

Contrast media extravasation in the stomach might be caused by heat or by an electrically induced mucosal defect due to high-voltage injury. No additional findings have been discussed in the literature. Further evaluation by forensic imaging magnetic resonance microscopy could be an option for future investigations [14]. 
Perihepatic fluid and subhepatic contrast media extravasation as an indicator of a probable heat-induced liver defect cannot be detected by autopsy. This aspect highlights the supporting nature of postmortem imaging in forensic investigation, thus leading to more detailed reporting and reconnoitering $[15,16]$.

Both hands of the subject were deemed as the entry points of the current, whereas the feet and the back were deemed as the exit points. These findings led to the suspicion of a cardiac pathway for the current [4]. Nevertheless, radiological correlations to the superficial burn mark at the back, or the so-called Lichtenberg figure, as detected by external inspection of the body, could not be observed. However, cutaneous marks might mimic electrical marks, even though they are not [17].

Moreover, victims of low voltage alternating current may have no inner electrical burns; this possibility makes the diagnosis of electrocution difficult. Specific evidence of electrocution in the internal organs is often lacking [5].

Therefore, distinguishing between autopsy findings with prior postmortem imaging [18], particularly PMMR, might help to clarify whether electrically induced injuries are present-even for low voltage incidents.

This case supports the suggestion that postmortem radiological investigation adds valuable information regarding possible electricity-induced lesions in a body.

\section{Conclusion}

Postmortem imaging - including PMCT and PMMR with and without contrast-is able to visualize electrocutionrelated injuries. These observations may support the future image interpretation of cases with electricity injuries in the living and the deceased.

This case emphasizes the value of postmortem imaging investigation in medico-legal issues.

\section{Key points}

1. PMMR is useful for evaluating skeletal muscle disorders.

2. Contrast media-enhanced PMCT detects mucosa defects in the stomach.

3. PMCTA detects liver defects that are macroscopically not visible.

4. PMCT and PMMR can visualize electrocution-related injuries.

5. Our findings may support further image interpretation.

\section{References}

1. Maghsoudi H, Adyani Y, Ahmadian N. Electrical and lightning injuries. J Burn Care Res Off Publ Am Burn Assoc. 2007;28: 255-61.

2. Ghavami Y, Mobayen MR, Vaghardoost R. Electrical burn injury: a five-year survey of 682 patients. Trauma Mon. 2014; 19(4):e18748.

3. Arnoldo BD, Purdue GF, Kowalske K, Helm PA, Burris A, Hunt JL. Electrical injuries: a 20-year review. J Burn Care Rehabil. 2004;25:479-84.

4. Saukko P, Knight B. Electrical fatalities. In: Saukko P, Knight B, editors. Knight's forensic pathology. London: Arnold; 2004. p. $326-38$.

5. Shetty BSK, Kanchan T, Acharya J, Naik R. Cardiac pathology in fatal electrocution. Burns. 2014;40:e45-6.

6. Kitulwatte I, Pollanen MS. Electrical injury of the neck and cardiac air embolism: a novel mechanism of death. Forensic Sci Med Pathol. 2008;5:32-5.

7. Bhatt DL, Gaylor DC, Lee RC. Rhabdomyolysis due to pulsed electric fields. Plast Reconstr Surg. 1990;86:1-11.

8. Ross S, Spendlove D, Bolliger S, Christe A, Oesterhelweg L, Grabherr S, et al. Postmortem whole-body CT angiography: evaluation of two contrast media solutions. Am J Roentgenol. 2008;190:1380-9.

9. Flach PM, Gascho D, Schweitzer W, Ruder TD, Berger N, Ross SG, et al. Imaging in forensic radiology: an illustrated guide for postmortem computed tomography technique and protocols. Forensic Sci Med Pathol. 2014;10:583-606.

10. Moratalla MB, Braun P, Fornas GM. Importance of MRI in the diagnosis and treatment of rhabdomyolysis. Eur $\mathrm{J}$ Radiol. 2008;65:311-5.

11. May DA, Disler DG, Jones EA, Balkissoon AA, Manaster BJ. Abnormal signal intensity in skeletal muscle at MR imaging: patterns, pearls, and pitfalls. Radiographics. 2000;20:295-315.

12. Fleckenstein JL, Chason DP, Bonte FJ, Parkey RW, Hunt JL, Purdue GF, et al. High-voltage electric injury: assessment of muscle viability with MR imaging and Tc-99 m pyrophosphate scintigraphy. Radiology. 1995;195:205-10.

13. Vale A. Rhabdomyolysis. Medicine (Baltimore). 2007;35:554-5.

14. Thali MJ, Dirnhofer R, Becker R, Oliver W, Potter K. Is, "virtual histology" the next step after the "virtual autopsy"? Magnetic resonance microscopy in forensic medicine. Magn Reson Imaging. 2004;22:1131-8.

15. Schulze C, Hoppe H, Schweitzer W, Schwendener N, Grabherr S, Jackowski C. Rib fractures at postmortem computed tomography (PMCT) validated against the autopsy. Forensic Sci Int. 2013;233:90-8.

16. Ruder TD, Germerott T, Thali MJ, Hatch GM. Differentiation of ante-mortem and post-mortem fractures with MRI: a case report. Br J Radiol. 2011;84:e75-8.

17. Byard RW. Infiltrating carcinoma of the breast mimicking electrocution. Forensic Sci Med Pathol. 2010;6:71-2.

18. Kawasumi Y, Usui A, Hosokai Y, Sato M, Funayama M. Heat haematoma: post-mortem computed tomography findings. Clin Radiol. 2013;68:e95-7. 